\title{
CRANIAL ULTRASOUND: EFFICIENT SCREENING TOOL FOR EARLY DETECTION OF BRAIN INJURY IN PRETERM INFANTS
}

\author{
Dini Rachma Erawati ${ }^{1}$, Yuyun Yueniwati ${ }^{1}$ \\ Correspondence: yuyun@ub.ac.id \\ ${ }^{l}$ Radiology Department, Faculty of Medicine, Universitas Brawijaya, Malang, Indonesia
}

\begin{abstract}
Article History:
Received: October 5, 2019

Accepted: September 29, 2020

Published: January 1, 2020

Cite this as:

Erawati DR, Yueniwati Y.

Cranial ultrasound: efficient screening tool for early detection of brain injury in preterm infants. Malang Neurology Journal; 2021.7:7-11.

http://dx.doi.org/10.21776/ub.mnj 2021.007.01.2
\end{abstract}

\section{ABSTRACT}

Background: Cranial ultrasound becomes an important diagnostic tool to evaluate brain injury in infants. Brain injury is a major complication for preterm birth. The brain injury of preterm infants differs from that of a term infant. Brain injury has correlation with gestational age and mode of delivery.

Objective: To analyze the correlation between cranial ultrasound findings with gestational age and mode of delivery and to reveal if cranial ultrasound can be used to detect brain injury in premature infants.

Methods: An observational analytic study using cross-sectional design took place in Saiful Anwar Hospital Malang, Indonesia. 38 healthy preterm infants underwent cranial ultrasound examination within the first four day of life. Fisher Exact test was used to analyze the correlation between cranial ultrasound findings with gestational age and mode of delivery.

Results: Most of the healthy preterm infants $(89.5 \%)$ were $\geq 32$ weeks gestational age, and $52.6 \%$ of samples had caesarean section as their mode of delivery. There were three abnormal findings in cranial ultrasound; increased periventricular echogenic (5.3\%), increased parenchym echogenic $(5.3 \%)$, and indistinguishable of gray-white matter differentiation $(5.3 \%)$. There was no significant correlation between abnormal cranial ultrasound findings with gestational age and mode of delivery $(\mathrm{p}=0.202 ; \mathrm{p}=0.218)$.

Conclusion: There were abnormal cranial ultrasound findings in some healthy preterm infants despite no significant correlation between ultrasound findings with gestational age and mode of delivery. Cranial ultrasound in preterm infants could become a screening tool for early detection of brain injury.

Keywords: Brain injury, cranial ultrasound, healthy preterm infants, gestational age, mode of delivery

\section{Introduction}

Preterm births remain as a major challenge in prenatal health. The World Health Organization (WHO) in 2018 stipulated that every year, an estimated 15 million babies are born preterm (before completing the 37 weeks of gestation), and this number is rising. ${ }^{1,2}$

Preterm birth complications are the leading cause of death among children under 5 years of age, responsible for approximately 1 million deaths in 2015 . Preterm births are the major cause of neonatal mortality and nearly half of all cases have had congenital neurological disorders including cerebral palsy. Although all births less than 37 weeks are classified as preterm births, the births less than 32 weeks' gestation comprise the highest mortality and neonatal disorders. $^{3,4}$

Brain injury is a major complication of preterm birth. It puts the infants that survive in conditions at risk of developmental disorders, cognitive dysfunction, and impaired manner. The brain injury of preterm infants differs from that of full-term infant. The most significant development differences that occur in the third semester pregnancy is the maturation of pressure-passive circulation, involution of the germinal matrix, selective stage-dependent differences in vascular white matter, and the sensitivity of oligodendrocyte against hypoxic injury. Cerebral injuries, such as white matter lesion and intra ventricular hemorrhage, are considered the cause of neurological disorders in preterm infants. ${ }^{5,6}$

A large number of prenatal risk factors with different capacities in terms of prediction of neurologic developmental disorders have been identified. Some multiple risk factors increase the risk of disability, which may act as additional effector multiplication effect. Some examples of prenatal risk factors are antepartum hemorrhage, congenital infections, preeclampsia, mode of delivery, short gestational age, microcephaly, and congenital anomalies. In addition, socio-economic status, parental education, maternal age, and parity also influence the outcome of the abnormality. $4,7,8$

Cranial ultrasound has become an important diagnostic tool since the 1970's. Its non-invasive nature has caused the ultrasound to become an ideal imaging modality in neonates. The fontanel and other structures of neonates and 
young infants are still open, thus allowing them to be the acoustic window for evaluating the brain. The cranial ultrasound examination has several advantages, among which are: minimal manipulation in infants, safe, repeatable so that it can evaluate the evolution maturation and lesions in the brain, and reliable in detecting brain pathology (such as hemorrhage, cystic lesions, ischemia, calcified, infection of the brain, and brain structures of major anomalies). In addition, the cranial ultrasound is relatively cheap compared to other neurological imaging modalities and is also a good modality for serial brain imaging for the neonates. 8,9

Preterm infants who are clinically healthy have the possibilities of having brain injury that could not be visualized on ultrasound examination. It means, the visible abnormalities on ultrasound sometimes do not provide significant clinical symptoms or are asymptomatic. Some research literature mentioned that in spite of full term or near-full term gestation and declared as healthy, infants could show an abnormality by cranial ultrasound imaging. ${ }^{10,11}$ Research by Mercuri et al in 1998 on healthy infants born with a gestational age between 36 and 42 weeks showed abnormalities in the cranial ultrasound examination in 35 out of 177 infants; mostly ischemic (periventricular and thalamus) and hemorrhagic lesions. ${ }^{12-14}$ Research by Hagmann et al in 2010 on healthy infants born full term in Uganda showed abnormalities presented by the cranial ultrasound examination in 55 from 112 infants; in the form of an increase in white matter echogenic, sub ependymal cysts, and choroid plexus cysts. ${ }^{13}$

Wang et al in 2004 found 6 from 2309 (0.26\%) full term healthy infants have major brain lesions, such as intra ventricular hemorrhage, intra parenchymal bleeding and corpus callosum agenesis. ${ }^{15}$

In order to prevent the missing prognostic abnormalities, a screening protocol in the form of cranial ultrasound examination should be conducted at least four or five times. The first examination should be conducted as early as possible in order to detect long-term intra-uterine damage or congenital malformations. It is important to be sure whether the results appear normal at this time, whether the anatomy looks normal for the infant's gestational age, whether there is not a picture of a congenital infection or metabolic disease, and to generate baseline data for management problems that could arise later, such as bleeding or echogenic of white matter. ${ }^{8,9}$

This study aimed to find the relationship between the cranial ultrasound findings with the gestational age and mode of delivery and to reveal if the cranial ultrasound can be used to detect brain injury in healthy preterm infants in Saiful Anwar Hospital Malang, Indonesia.

\section{Methods}

This study was an observational-analytic with a crosssectional design, conducted in the perinatology and ultrasound room of the Saiful Anwar Hospital Malang. The protocol has been approved by the Medical Research Ethics Committee of the Medical Faculty of Universitas Brawijaya Malang No: 352/KEPK/VII/2012. A total of 38 healthy preterm infants were included in this study. The inclusion criteria of the study subjects were healthy preterm infants at the age of $\leq 4$ days. Infants who have congenital abnormalities were excluded from the study.

Medical records and anamnesis were used to collect data on gestational age and mode of delivery. The cranial ultrasound was performed by three radiologists who have experiences in their field for at least 10 years. In order for the diagnosis to be positive, a minimum of two examiners statements is needed. The examination was performed using an ultrasound (General Electric Logic S6 series, USA) with a curved transducer $(5 \mathrm{MHz})$ and linear transducer $(10 \mathrm{MHz})$ and was conducted with the anterior fontanel window and six coronal pieces and five sagittal pieces. Relationships between the gestational age and mode of delivery with a cranial ultrasound imaging were tested using the Fisher Exact test with a 95\% confidence level, $\alpha=$ 0.05 significant when $\mathrm{p}<0.05$.

\section{Results}

Out of the 38 preterm infants, $52.6 \%$ of them were female and $47.4 \%$ were male. A total of $52.6 \%$ of the infants were born through caesarian section. Most of the infants were $\geq$ 32 weeks $(89.5 \%)$ and had birth weight between 1500 and 2500 grams.

Cranial ultrasound examination showed three kinds of abnormal imaging, which were the increased periventricular echogenic, increased parenchymal echogenic, and indistinguishable gray-white matter differentiation (Table $1)$.

Table 1. Cranial ultrasound results

\begin{tabular}{l|l}
\hline & \multicolumn{1}{c}{$\mathbf{n}(\%)$} \\
\hline Periventricular echogenic & \\
Increased & $2(5,3)$ \\
Not increased & $36(94,7)$ \\
Parenchymal echogenic & \\
$\quad$ Increased & $2(5,3)$ \\
$\quad$ Not increased & $36(94,7)$ \\
White-gray matter differentiation & \\
Indistinguishable & $2(5,3)$ \\
Distinguishable & $36(94,7)$ \\
\hline
\end{tabular}

Two infants revealed increased periventricular echogenic (5.3\%); one infant with an increase in the right lateral superior periventricular and the other one in the caudothalamic notch (Figure 1).

Two infants were found to have increased parenchymal echogenic (unilateral and focal thalamus echogenic) (5.3\%) (Figure 2).

The indistinguishable differentiation of gray-white matter was discovered in two infants $(5.3 \%)$. Distinguishing between the cortical and sub-cortical was difficult to perform in both infants (Figure. 3).

It was found that in 34 infants with a gestational age $\geq 32$ weeks, $3 \%$ of them had an increase in periventricular echogenic, while in infants with gestational age $<32$ weeks, $25 \%$ of them had it. Increased parenchymal echogenic was found in $6 \%$ of infants with gestational age $\geq 32$ weeks. The indistinguishable gray-white matters were found as much as $3 \%$ in the gestational age group $\geq 32$ weeks and $25 \%$ in the gestational age group $<32$ weeks. There were differences in the proportion of each variable of cranial ultrasound based on the gestational age, but the differences were not statistically significant $(\mathrm{p}>0.05)$ 
Table 2. The results of cranial ultrasound based on the gestational age

\begin{tabular}{|c|c|c|c|c|c|c|c|c|c|}
\hline \multirow[b]{2}{*}{$\begin{array}{l}\text { Gestational } \\
\text { age }\end{array}$} & \multicolumn{3}{|c|}{ Periventricular echogenic } & \multicolumn{3}{|c|}{ Parenchymal echogenic } & \multicolumn{3}{|c|}{ Gray-white matter differentiation } \\
\hline & $\begin{array}{l}\text { Increased } \\
\Sigma(\%)\end{array}$ & $\begin{array}{l}\text { Not } \\
\text { Increased } \Sigma \\
(\%)\end{array}$ & $\mathrm{p}$ & $\begin{array}{l}\text { Increased } \\
\Sigma(\%)\end{array}$ & $\begin{array}{l}\text { Not Increased } \\
\Sigma(\%)\end{array}$ & $\mathrm{p}$ & $\begin{array}{l}\text { Indistinguishable } \\
\Sigma(\%)\end{array}$ & $\begin{array}{l}\text { Distinguishable } \Sigma \\
\text { (\%) }\end{array}$ & $\mathrm{p}$ \\
\hline$<32$ weeks & $1(25)$ & $3(75)$ & & $0(0)$ & $4(100)$ & 0,798 & $1(25)$ & $3(75)$ & 0,202 \\
\hline 232 weeks & $1(3)$ & $33(9)$ & 0,202 & $2(6)$ & $32(94)$ & 0,198 & $1(3)$ & $33(97)$ & 0,202 \\
\hline
\end{tabular}

Table 3. The results of cranial ultrasound based on the mode of delivery

\begin{tabular}{|c|c|c|c|c|c|c|c|c|c|}
\hline \multirow[b]{2}{*}{$\begin{array}{l}\text { Mode of } \\
\text { delivery }\end{array}$} & \multicolumn{3}{|c|}{ Periventricular echogenic } & \multicolumn{3}{|c|}{ Parenchymal echogenic } & \multicolumn{3}{|c|}{ Gray-white matter differentiation } \\
\hline & $\begin{array}{l}\text { Increased } \\
\Sigma(\%)\end{array}$ & $\begin{array}{l}\text { Not } \\
\text { Increased } \Sigma \\
(\%)\end{array}$ & $\mathrm{p}$ & $\begin{array}{l}\text { Increased } \\
\Sigma(\%)\end{array}$ & $\begin{array}{l}\text { Not } \\
\text { Increased } \\
\Sigma(\%)\end{array}$ & $\mathrm{p}$ & $\begin{array}{l}\text { Indistinguishable } \Sigma \\
\text { (\%) }\end{array}$ & $\begin{array}{l}\text { Distinguishable } \\
\Sigma(\%)\end{array}$ & $\mathrm{p}$ \\
\hline Spontaneous & $2(11)$ & $16(89)$ & \multirow[b]{2}{*}{0,218} & $0(0)$ & $18(100)$ & \multirow[b]{2}{*}{0,270} & $2(11)$ & $16(89)$ & \multirow[b]{2}{*}{0,218} \\
\hline $\begin{array}{l}\text { Section } \\
\text { caesarean }\end{array}$ & $0(0)$ & $20(100)$ & & $2(10)$ & $18(90)$ & & $0(0)$ & $20(100)$ & \\
\hline
\end{tabular}

Significant $p<0.05$

Table 4. The results of cranial ultrasound based on the combination of mode of delivery and gestational age

\begin{tabular}{|c|c|c|c|c|c|c|c|c|c|}
\hline & \multicolumn{3}{|c|}{ Periventricular echogenic } & \multicolumn{3}{|c|}{ Parenchymal echogenic } & \multicolumn{3}{|c|}{ Gray white matter differentiation } \\
\hline & $\begin{array}{l}\text { Increased } \\
\Sigma(\%)\end{array}$ & $\begin{array}{l}\text { Not } \\
\text { Increased } \Sigma \\
(\%)\end{array}$ & $\mathrm{p}$ & $\begin{array}{l}\text { Increased } \\
\Sigma(\%)\end{array}$ & $\begin{array}{l}\text { Not } \\
\text { Increased } \\
\Sigma(\%) \\
\end{array}$ & $\mathrm{p}$ & $\begin{array}{l}\text { Indistinguishable } \\
\Sigma(\%)\end{array}$ & $\begin{array}{l}\text { Distinguishable } \Sigma \\
(\%)\end{array}$ & $\mathrm{p}$ \\
\hline $\begin{array}{l}\text { Spontaneous } \\
\text { and } \geq 32 \\
\text { weeks }\end{array}$ & $1(7,1)$ & $13(92,9)$ & \multirow{3}{*}{0,114} & $0(0)$ & $14(100)$ & \multirow{3}{*}{0,387} & $1(7,1)$ & $13(92,9)$ & \multirow{3}{*}{0,114} \\
\hline $\begin{array}{l}\text { Spontaneous } \\
\text { and < } 32 \\
\text { weeks }\end{array}$ & $1(25)$ & $3(75)$ & & $0(0)$ & $4(100)$ & & $1(25)$ & $3(75)$ & \\
\hline $\begin{array}{l}\text { Section } \\
\text { caesarean and } \\
\geq 32 \text { weeks }\end{array}$ & $0(0)$ & $20(100)$ & & $2(10)$ & $18(90)$ & & $0(0)$ & $20(100)$ & \\
\hline
\end{tabular}

Significant $p<0.05$

It was found that in 18 infants with spontaneous birth, $11 \%$ of them had increased periventricular echogenic. On the other hand, there was no such image found from the 20 infants with caesarean section birth. No increase in parenchymal echogenic was present on the infant group with spontaneous birth. The white gray matter differentiation was found in $10 \%$ infants with spontaneous birth. However, it was not discovered in the group of infants with caesarean section birth. There were differences in the proportion of each variable of cranial ultrasound based on the mode of delivery, but the differences were not statistically significant $(\mathrm{p}>0.05)$.

Statistical analysis showed that there is no significant correlation between the results of cranial ultrasound with the combination of gestational age and mode of delivery ( $\mathrm{p}$ $>0.05)$.

\section{Discussion}

The The samples obtained in this study were 38 preterm infants, 18 of whom were male and 20 were female. There were 34 infants with gestational age $\geq 32$ weeks and 4 infants with gestational age $<32$ weeks. Macones stated that birth at less than 32 gestational weeks has the highest mortality and neonatal disorders. After 32 gestational weeks, there was a noticeable increase in vascular system and the number of anastomosis in the deep white matter, and thus changing the pattern of injury. 3,16
A total of 18 infants were delivered spontaneously, and 20 infants were born through caesarean section. Previous study showed that birth by emergency cesarean, with or without a prior attempt of vaginal births, correlated with high intracranial hemorrhage compared to spontaneous birth, whereas the incidence of brain hemorhage in elective cesarean did not differ significantly with the spontaneous births.

This research found two infants with an increased periventricular echogenic: one infant with $\geq 32$ gestational weeks with a lesion in the lateral superior ventricles, and another infant with a gestational age $<32$ weeks with a lesion in the caudo-thalamic notch with ventricular asymmetry. Both underwent spontaneous delivery. It is likely for a preterm infant with a hyper-echoic lesion in the periventricular to have a white matter injury, but edema can also present such image and show improvements without any further brain damage. ${ }^{3,17}$ Increased periventricular echogenic might show a normal condition if it lasts less than a week. Infants with increased echogenic in the caudothalamic notch require further observation because the lesions in this area are probably a germinal matrix hemorrhage which usually occurs in preterm infants. ${ }^{18}$ 


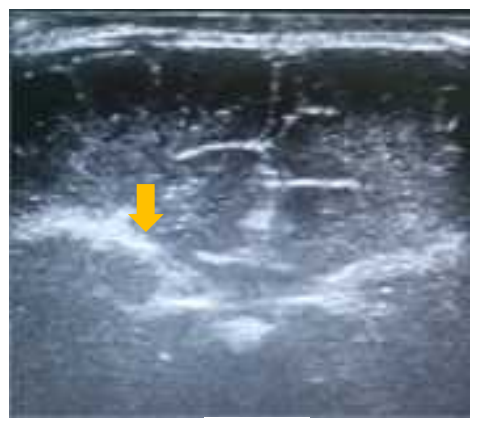

(A)

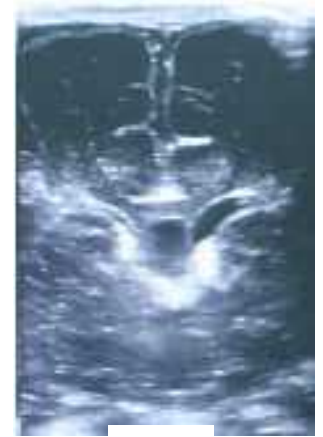

(B)
Figure 1. USG examination result with increased echogenic A) Right Anterior Periventricular (yellow arrow) is more increased than the left side, B) periventricular (caudothalamic notch)

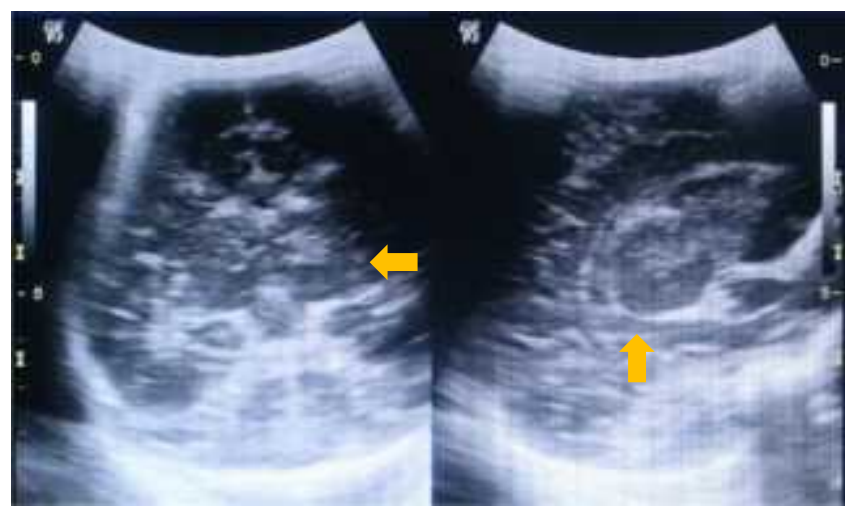

Figure 2. USG examination result with increased unilateral echogenic and focal in left thalamus (yellow arrow)

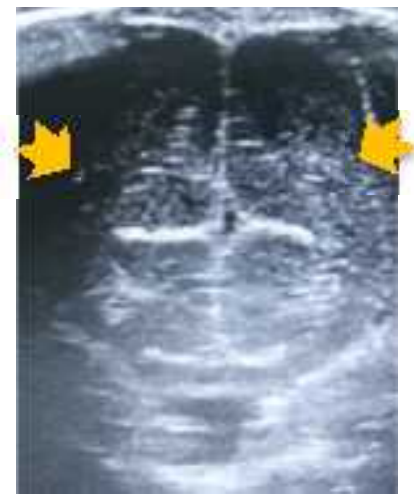

Figure 3. Indistinguishable differentiation of gray-white matter (yellow arrow)

Two infants had unilateral increased echogenic in the thalamus. The density of unilateral thalamus in preterm infants was reported by de Vries (1992) and van WezelMeijlrin (1999) and it is related to severe asphyxia condition and treatment of longer ventilation, although the prognosis is better than infants who have bilateral lesions of the thalamus. ${ }^{13}$ However, the studies on healthy full-term infants in Uganda found that unilateral lesions of the thalamus also occur in asymptomatic infants. This study found two infants had increased unilateral and focal thalamus echogenic, in which both the infants had a gestational age $\geq 32$ weeks with caesarean section mode of delivery. Both may not be related to global hypoxic ischemic. Infarction in the territory of the perforator artery can explain these ultrasound findings, because the perforator artery is not always associated with acute symptoms. Unilateral abnormalities can also be thalamus hemorrhage, which is associated with venous thrombosis, usually accompanied with intra ventricular hemorrhage and clinical seizures in the patient. This study did not find any intra ventricular hemorrhage image in the ultrasound findings. ${ }^{13,14}$

The indistinguishable gray-white matter was found in one infant in each gestational age group $\geq 32$ weeks and $<32$ weeks. Both infants were delivered spontaneously. The visualized cortical structures became more hyper-echoic, making it difficult to distinguish from the subcortical structures. The edema conditions could give such image and will improve without any further brain damage. In this study, there was no increased intracranial pressure in all infants. ${ }^{7,19}$

Statistically, there was no significant correlation between abnormal ultrasound findings (increased periventricular echogenic, increased parenchymal echogenic, and graywhite matter differentiation) with the gestational age and mode of delivery. A similar studies on full term infants with more samples also showed that there was no correlation between ultrasound findings with gestational age and mode of delivery The research conducted by Behnke et al 1999 on 266 infants with cranial ultrasound examination within 96 hours of birth age found that there was no significant correlation between neurobehavior status with head ultrasound abnormalities findings. Therefore, it was concluded that ultrasound abnormalities found within 96 hours of life have no clinical significance in the neonatal period. The data in this study was obtained within 4 days, and therefore it is still possible that the cranial ultrasound findings are light findings and do not affect the clinical condition of the infants. ${ }^{5,14}$

\section{Conclusion}

There are abnormal cranial ultrasound findings in some healthy preterm infants, although there is no significant correlation between ultrasound findings with gestational age and mode of delivery. Cranial ultrasound in preterm infants can become a screening tool for an early detection of brain injury.

\section{Acknowledgement}

This work was supported by grants from Faculty of Medicine, Universitas Brawijaya, Malang, Indonesia

\section{References}

1. Blencowe H, Cousens S, Chou D, Oestergaard M, Say L, Moller AB, et al. Born Too Soon: The global epidemiology of 15 million preterm births. Reprod Health; 2013. DOI : 10.1186/1742-4755-10-S1-S2

2. WHO. Newborns: reducing mortality. World Health Organization. 2018.

3. Hinojosa-Rodríguez M, Harmony T, Carrillo-Prado C, Van Horn JD, Irimia A, Torgerson C, et al. Clinical neuroimaging in the preterm infant: Diagnosis and prognosis. NeuroImage: Clinical; 2017.

DOI: 10.1016/j.nicl.2017.08.015 
4. Ecury-Goossen GM, Camfferman FA, Leijser LM, Govaert P, Dudink J. State of the art cranial ultrasound imaging in neonates. J Vis Exp; 2015.

DOI: $10.3791 / 52238$

5. De Vries LS, Benders MJNL, Groenendaal F. Progress in Neonatal Neurology with a Focus of Neuroimaging in the Preterm Infant. Neuropediatrics; 2015.

DOI: $10.1055 / \mathrm{s}-0035-1554102$

6. Pisani F, Spagnoli C. Monitoring of newborns at high risk for brain injury. Italian Journal of Pediatrics; 2016. Avalaible from:

https://ijponline.biomedcentral.com/articles/10.1186/s1 3052-016-0261-8

7. Yueniwati Y, Wangsadjaja C, Yulidani II, Rianawati SS, Al Rasyid H. The Role of Brain Magnetic Resonance Imaging (MRI) as an Early Detector of Cognitive Impairment. J Neurosci Rural Pr; 2018. 9(3):350-3. DOI: 10.4103/jnrp.jnrp_542_17

8. Doria V, Arichi T, David Edwards A. Magnetic Resonance Imaging of the Preterm Infant Brain. Curr Pediatr Rev; 2014.

DOI: $10.2174 / 157339631001140408120821$

9. Van Den Broek NR, Jean-Baptiste R, Neilson JP. Factors associated with preterm, early preterm and late preterm birth in Malawi. PLoS One; 2014.9. DOI: 10.1371/journal.pone.0090128

10. Wezel-Meijler G, de Vries LS. Cranial Ultrasound Optimizing Utility in the NICU. Curr Pediatr Rev; 2014. DOI: 10.2174/157339631001140408120106

11. Hagberg H, David Edwards A, Groenendaal F. Perinatal brain damage: The term infant. Neurobiol Dis; 2016. DOI: 10.1016/j.nbd.2015.09.011

12. Robertson NJ, Hagmann CF, Acolet D, Allen E, Nyombi N, Elbourne D, et al. Pilot randomized trial of therapeutic hypothermia with serial cranial ultrasound and 18-22 month follow-up for neonatal encephalopathy in a low resource hospital setting in Uganda: study protocol. Trials; 2011;12:138.

DOI: $10.1186 / 1745-6215-12-138$

13. Hagmann CF, Robertson NJ, Acolet D, Chan D, Onda $\mathrm{S}$, Nyombi N, et al. Cranial ultrasound findings in well newborn Ugandan infants. Arch Dis Child Fetal Neonatal Ed; 2010.95:F338-44. DOI: $10.1136 /$ adc.2009.174607

14. Tann CJ, Nakakeeto M, Hagmann C, Webb EL, Nyombi N, Namiiro F, et al. Early cranial ultrasound findings among infants with neonatal encephalopathy in Uganda: An observational study. Pediatr Res; 2016. DOI: $10.1038 /$ pr.2016.77

15. Shukla R. Paediatric radiology. In: Final FRCR 2B Viva: A Survival Guide; 2011. ISBN: 9780521183079

16. Fenton TR, Kim JH. A systematic review and metaanalysis to revise the Fenton growth chart for preterm infants. BMC Pediatr; 2013. Avalaible from: https://bmcpediatr.biomedcentral.com/articles/10.1186/ 1471-2431-13-59

17. Bano S, Chaudhary V, Garga UC. Neonatal hypoxicischemic encephalopathy: A radiological review. Journal of Pediatric Neurosciences; 2017. Avalaible from: http://www.pediatricneurosciences.com

18. Yueniwati Y, Erawati DRDR. Head ultrasound findings in healthy preterm infants: Their correlation with gestational age and mode of delivery. J Neurol Sci [Internet]; 2013. Oct [cited 2014 Oct 19];333:e585. Available from: http://www.jnsjournal.com/article/S0022510X13023538/fulltext

19. Yueniwati Y, Wangsadjaja C. Fazekas scale evaluation on head MRI as a predictor of declined cognitive function. J Neurol Sci [Internet]; 2017. Dec 14;381:1033. DOI: 10.1016/j.jns.2017.08.2916 